\title{
von Kármán Vortex Street within an Impacting Drop
}

\author{
Marie-Jean Thoraval, ${ }^{1}$ Kohsei Takehara, ${ }^{2}$ Takeharu Goji Etoh, ${ }^{2}$ Stéphane Popinet, ${ }^{3}$ Pascal Ray, ${ }^{4}$ \\ Christophe Josserand, ${ }^{4}$ Stéphane Zaleski, ${ }^{4}$ and Sigurdur T. Thoroddsen ${ }^{1, *}$ \\ ${ }^{1}$ Division of Physical Sciences and Engineering \& Clean Combustion Research Center, \\ King Abdullah University of Science and Technology (KAUST), Thuwal, 23955-6900, Saudi Arabia \\ ${ }^{2}$ Department of Civil and Environmental Engineering, Kinki University, Higashi-Osaka, Japan \\ ${ }^{3}$ National Institute of Water and Atmospheric Research, Kilbirnie, Wellington 6003, New Zealand \\ ${ }^{4}$ Institut Jean Le Rond D'Alembert, UMR 7190, Université Pierre et Marie Curie, Paris, France
}

(Received 11 February 2012; published 29 June 2012)

\begin{abstract}
The splashing of a drop impacting onto a liquid pool produces a range of different sized microdroplets. At high impact velocities, the most significant source of these droplets is a thin liquid jet emerging at the start of the impact from the neck that connects the drop to the pool. We use ultrahigh-speed video imaging in combination with high-resolution numerical simulations to show how this ejecta gives way to irregular splashing. At higher Reynolds numbers, its base becomes unstable, shedding vortex rings into the liquid from the free surface in an axisymmetric von Kármán vortex street, thus breaking the ejecta sheet as it forms.
\end{abstract}

DOI: 10.1103/PhysRevLett.108.264506

Liquid drop splashing is part of our daily lives, from the morning shower to natural rain $[1,2]$. While it has been studied for more than one hundred years [3], it is only recently that advances in high-speed imaging techniques [4,5] have revealed its early dynamics [6,7]. Splashing refers herein to the breakup of a drop into smaller droplets during impact. Understanding the underlying mechanism that produces the smallest droplets is important, for example, for the number of microscopic aerosols which remain when those satellite droplets evaporate. Such aerosols affect human health and can act as nucleation sites during cloud formation.

For high-speed drop impact on a liquid pool, the ejecta sheet is the first stage leading to splashing. It was first observed in the inviscid numerical simulations of Weiss and Yarin [8] and in the experiments of Thoroddsen [9]. When the drop impacts at higher velocity, the speed of these ejecta sheets increases and they become thinner. The radial stretching of the sheets reduces their thickness even further, and they can remain intact even at thicknesses well under a micron [6]. When they eventually rupture, they can produce a myriad of very fine spray droplets. However, this mechanism does not continue for ever; at a critical Reynolds number, the smooth ejecta gives way to a more random splashing, which counterintuitively may produce fewer small droplets.

To understand the mechanisms leading from continuous ejecta sheets to irregular splashing, a systematic study of the early dynamics was conducted with ultrahigh-speed

Published by the American Physical Society under the terms of the Creative Commons Attribution 3.0 License. Further distribution of this work must maintain attribution to the author(s) and the published article's title, journal citation, and DOI.
PACS numbers: 47.32.ck, 47.20.Dr, 47.20.Ma, 47.55.D-

video imaging, over a range of impact velocities $U$, liquid viscosities $\mu$, and droplet diameters $D$ [10]. Figure 1 shows a classification of the results in terms of Reynolds number $\operatorname{Re}=\rho D U / \mu$, where $\rho$ is the liquid density, and splashing parameter $K$, which relates to the Weber number $\mathrm{We}=$ $\rho D U^{2} / \sigma$, where $\sigma$ is the surface tension, as $K=\mathrm{We} \sqrt{\mathrm{Re}}$.

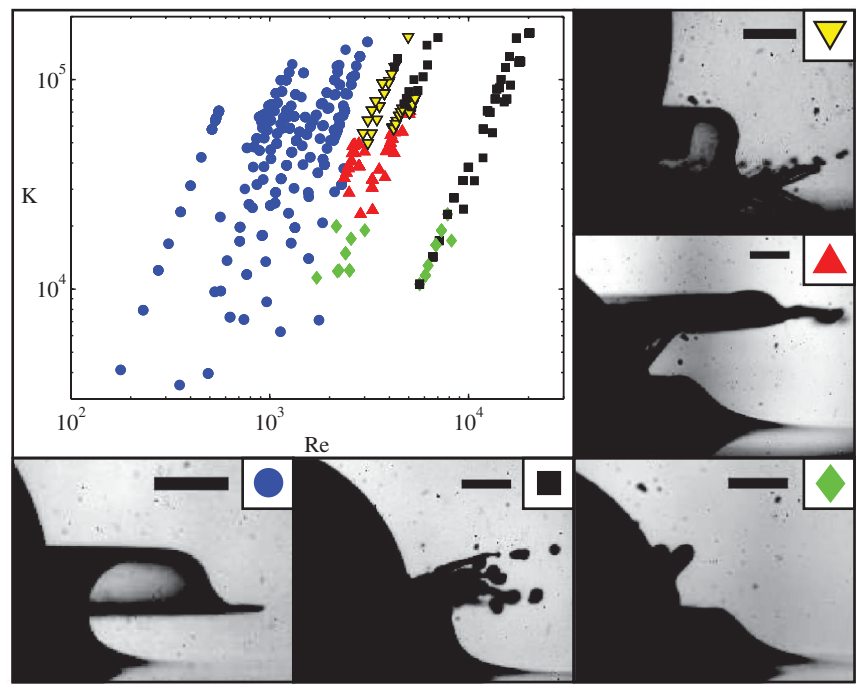

FIG. 1 (color). Characterization of the ejecta regimes. (O) Smooth ejecta sheet $\left(t=145 \mu \mathrm{s}, \operatorname{Re}=1410, K=5.62 \times 10^{4}\right)$. (ם) Irregular splashing $\left(t=360 \mu \mathrm{s}, \mathrm{Re}=1250, K=7.11 \times 10^{4}\right)$. (V) Bumping $\left(t=180 \mu \mathrm{s}, \quad \operatorname{Re}=3550, \quad K=7.44 \times 10^{4}\right)$. (A) Quartering $\left(t=630 \mu \mathrm{s}, \mathrm{Re}=2810, K=3.86 \times 10^{4}\right)$. $(\diamond)$ Protrusions rising up along the side of the drop $(t=$ $\left.630 \mu \mathrm{s}, \operatorname{Re}=2410, K=1.48 \times 10^{4}\right)$. The scale bars are all $500 \mu \mathrm{m}$ long. 
We are interested here in the higher $K$ regime, where splashing occurs [11-13].

The classification in Fig. 1 focuses on the ejecta shapes. In the lower range of Re (more viscous liquids), a smooth ejecta sheet emerges between the drop and the pool (O). However, in the highest range of Re, isolated droplets emerge from the neck, followed by a disturbed liquid surface, and no coherent ejecta can be identified; i.e., irregular splashing occurs ( $\mathbf{\square})$. In the intermediate regime $(\operatorname{Re} \approx 2000-6000)$, the ejecta sheets show a large variety of repeatable shapes. We have grouped them into 3 classes. At lower $K$ (lower impact velocities, $\diamond$ ), surface tension prevents the formation of an ejecta sheet. However, we observe some protrusions traveling up along the side of the drop, without ejection of droplets outwards [7]. At higher $K(\mathbf{\Delta})$, the ejecta sheet is more developed; however, it stays attached to the drop, stretching the ejecting sheet between the expanding tip of ejecta and the drop entering the pool. This regime is called quartering. This stretching can lead to the explosive rupturing of the sheet, which generates fast droplets of a large range of sizes through slingshot [6], by surface tension pulling on a free liquid sheet. In the upper range of $K(\boldsymbol{\nabla})$ we observe an intriguing phenomenon where the freestanding sheet interacts strongly with the downward-moving drop surface. This is shown in the sequence of Fig. 2(a), referred to as the bumping of the ejecta. The ejecta is strongly bent by the drop, and then folds at its apex. Overall snapshot of a bumping ejecta was included in Ref. [5] [their Fig. 8(c)].

Those experimental results clearly show the effect of the Reynolds number on the transition toward irregular splashing. Moreover, the results show new dynamics of the ejecta sheet interacting with the drop. This suggests that those interactions could underlie the irregular splashing.

To test this idea, we have chosen to reproduce the impact by numerical simulations. It is only recently that numerical simulations managed to identify the ejecta sheet $[8,14,15]$, because of the extreme range of scales involved and the challenges of interfacial flow simulations [16]. The intricate shapes observed herein were beyond reach in previous studies. We use the freely available code GERRIS [17-19] for its high parallelization and dynamic adaptive grid refinement, which allow us for the first time to reach enough precision to fully resolve the dynamics of the ejecta. This code uses the volume-of-fluid method to solve the incompressible Navier-Stokes equations. Furthermore, we start the simulation before impact, thus capturing the air-cushioning effect (see Ref. [10,20-25]).

Axisymmetric simulations faithfully reproduced all of the experimentally observed features, as we demonstrate in Fig. 2 for the bumping case. The shape of the drop in the simulation is perfectly spherical, ruling out the hypothesis that small deviations from spherical drop shapes in the experiments could be responsible for the drop interaction with the ejecta sheet.

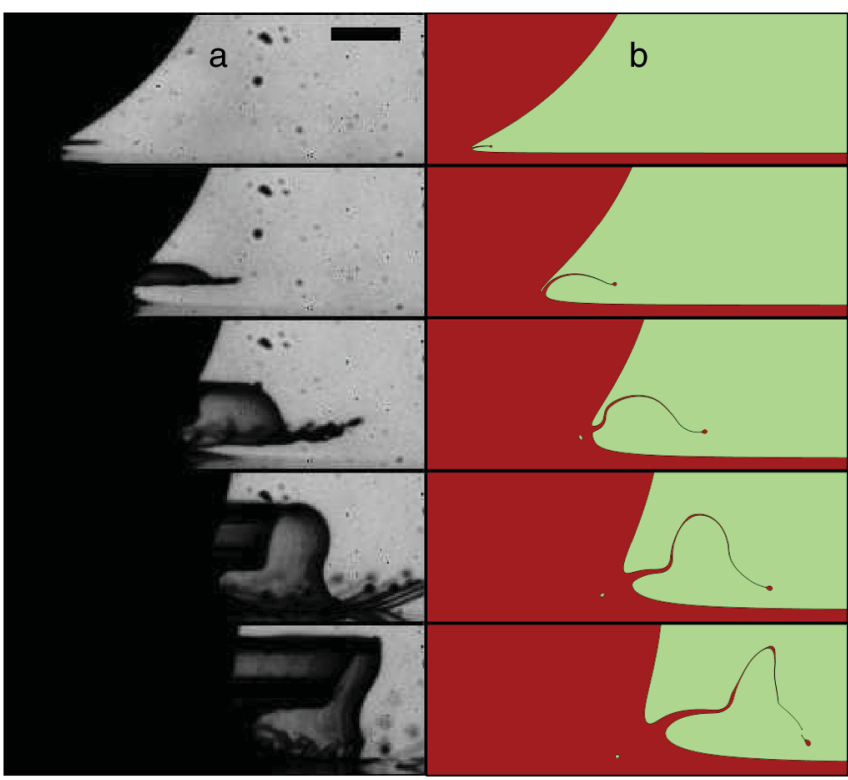

FIG. 2 (color). Comparison between experiment and axisymmetric numerical simulation for a bumping case. $U=4.04 \mathrm{~m} / \mathrm{s}$, $D=4.6 \mathrm{~mm}, \operatorname{Re}=3.55 \times 10^{3}, K=7.44 \times 10^{4}$. From top to bottom, observations at time 30, 80, 130, 180, and $230 \mu \mathrm{s}$ after contact. (a) Experimental observation. The static dark points correspond to dust on the camera sensor. The video was taken at 200000 frames per second. (b) Numerical simulation of the drop impact for exactly the same times after impact, same scale and same field of view as in the experiment presented in (a). In the last image, the leading part of the ejecta sheet becomes smaller than the grid size by stretching between the apex and the tip and thus breaks into nonphysical droplets. The axisymmetric simulations cannot include the threedimensional effects, such as the breakup of the tip observed in (a). The scale bar is $500 \mu \mathrm{m}$ long. Supplemental videos show the two evolutions [10].

To study this transition to irregular splashing we increase $\mathrm{Re}$, while keeping $K$ constant, from a smooth ejecta sheet [Fig. 1(@)] to irregular splashing (ם). This was done for two different $K$ values, corresponding to the bumping $(\boldsymbol{\nabla})$ and quartering $(\boldsymbol{\Delta})$ regimes.

The position of the base of the ejecta $r_{K}$ [Figs. 3(a) and 3(b)] follows very closely the geometric relation predicted by Josserand and Zaleski [14] $r_{K}=C r_{J}$, independent of $\mathrm{Re}$, where $r_{J}$ is the radius where an unperturbed drop would meet the original pool surface. A simple geometric model [6] suggested that $\theta$ increases as $\theta \sim \sqrt{t^{*}}$, where $t^{*}$ is the time nondimensionalized by $\tau=D / U$, whereas the simulations show that $\theta$ grows linearly before bumping [Fig. 3(c)]. However, the ejecta rises faster for higher Re. The collapse of the curves in Fig. 3(d) shows that $\theta$ grows at a rate proportional to $\sqrt{\mathrm{Re}}$. The angle of the ejectionvelocity vector at the middle of the base also follows a similar trend, and increases proportionally to $\sqrt{\mathrm{Re}}$.

At lower Re, $\theta$ increases slowly enough for the ejecta to escape the drop. However, from $\operatorname{Re} \gtrsim 3000$, the ejecta 
a
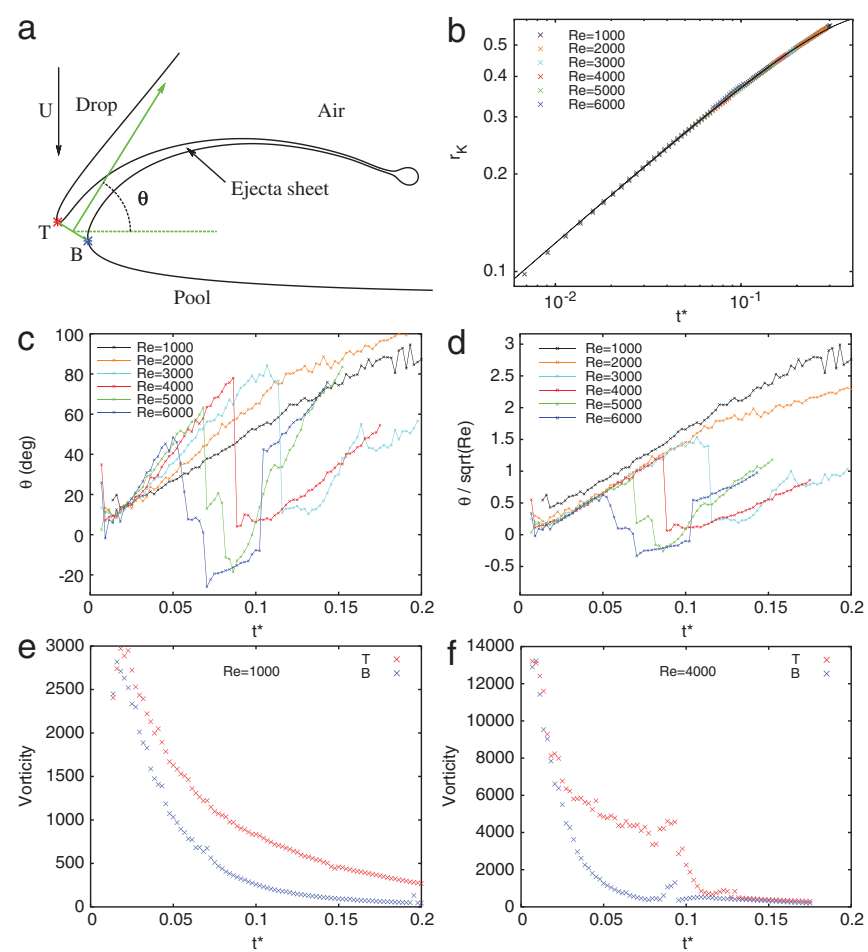

FIG. 3 (color). Evolution of the base of the ejecta sheet with $\operatorname{Re}$ at $K=7.44 \times 10^{4}$. Quantities are nondimensionalized by the drop diameter $D$, the drop impact velocity $U$, and the drop entry time $\tau=D / U$. (a) Definition sketch. The base of the ejecta sheet is defined as the segment between the two points of maximum curvature of the interface ( $T$ on the drop side and $B$ on the side of the pool). The angle of the ejecta sheet $\theta$ is the angle between the horizontal and the normal to the base. (b) Evolution of the ejecta base radial position $r_{K}$, defined as the distance from the axis of symmetry to the middle of $T B$ in (a), vs the nondimensional time $t^{*}=t / \tau$, for Re from 1000 to 6000 . The solid curve is $1.23 r_{J}$, where $r_{J}=\sqrt{t^{*}\left(1-t^{*}\right)}$ is the radius where an undisturbed drop would meet the pool. (c) Evolution of $\theta$ (in degrees) for Re from 1000 to 6000 . The sharp drops correspond to bumping events, as the position of $T$ suddenly moves up along the drop side. (d) Same curves as (c), where the angle is scaled by $\sqrt{\mathrm{Re}}$. (e),(f) Evolution of the maximum positive vorticity (red) and maximum absolute negative vorticity (blue) in the liquid near the ejecta base for $\operatorname{Re}=1000$ (e) and $\operatorname{Re}=4000$ (f). The positive maximum is located near $T$, and the negative maximum near $B$.

sheet rises too fast, thus impacting the drop surface. The resulting bumping sharply decreases $\theta$. This interaction of the drop and the ejecta sheet observed experimentally occurs earlier at higher Reynolds numbers, eventually breaking the ejecta sheet. This is consistent with the interpretation that this interaction is responsible for the irregular splashing observed at higher Re.

Vorticity also plays an important role in the dynamics of the ejecta sheet [Figs. 3(e) and 3(f)]. For a stationary twodimensional free surface, vorticity is generated at the free surface proportionally to the interface curvature $\kappa$ and the tangential flow velocity $q$ : $\omega=2 \kappa q$ (see for instance Ref. [26] 5.14, and [27,28] for low We drop impacts). This vorticity is then diffused into a thin boundary layer, which can separate to enter the liquid. Numerical simulations indeed show concentrated vorticity near points $T$ and $B$ at the base of the ejecta as the flow moves faster around the highly curved base to enter the ejecta (Fig. 4). At the early stage of the ejecta formation, both sides of the base produce a similar strength of vorticity. This initial vorticity scales as $\sqrt{\mathrm{Re}}$ as observed previously [14]. However, the difference in vorticity (absolute values) between the two sides increases initially linearly with time, before decreasing again. Moreover, this difference is higher for larger $\mathrm{Re}$ [Fig. 3(f)].

By looking closely at the neck region during the impact, we can identify fundamental changes in the vorticity structure as Re is increased (Fig. 4). Note that in Fig. 4(a) most of the liquid in the sheet originates from the pool, in agreement with dye visualizations [9]. For the lower range of Re [Figs. 4(a) and 4(d)], the vorticity stays concentrated near the free surface at the neck of the ejecta sheet. As there is stronger vorticity generated at the top of the ejecta base, a vorticity layer of one sign separates the drop and the pool liquids but it remains stable. $K$ affects the shape of the outer part of the ejecta sheet, as we observe by comparing Figs. 4(a) and 4(d), consistently with experimental observations [6]. For intermediate $\mathrm{Re}$, the interface remains stable in its early evolution. In the bumping case (b), the rising ejecta sheet contacts the downward-moving drop surface. This creates a shear instability, generating a toroidal vortex structure around the entrapped bubble. In the quartering case (e), the ejecta sheet leaves the neck region to climb up the drop, pulled by higher surface tension. This also creates a shear instability between the climbing liquid from the pool and the drop liquid moving down, forming a row of vortex rings of the same sign. These vortices near the free surface leave their signature [29] by creating waves below the rising sheet, a feature also observed experimentally (Fig. 1) [7]. However, all such vortical effects are absent from inviscid theory and simulations [8,30].

At even higher Re [Figs. 4(c) and 4(f)], vorticity is shed behind the base of the ejecta sheet, in a way reminiscent of the von Kármán vortex street, here forming alternatingsign vortex rings. For the first 7 shedding cycles, the local Reynolds number based on the radial speed and width of the neck takes value around 70 and the Strouhal number $\mathrm{St}=f D / U$ is around $0.11 \pm 0.05$, in good agreement with related Kármán streets. This analogy with the vortex shedding of a cylinder suggests that vorticity can be responsible for the oscillations of the base [31] and would be present even without them. However, those oscillations will amplify the vorticity difference between the two sides of the base through surface curvature, reinforcing the oscillations and the separation of individual vortices. Therefore, we 


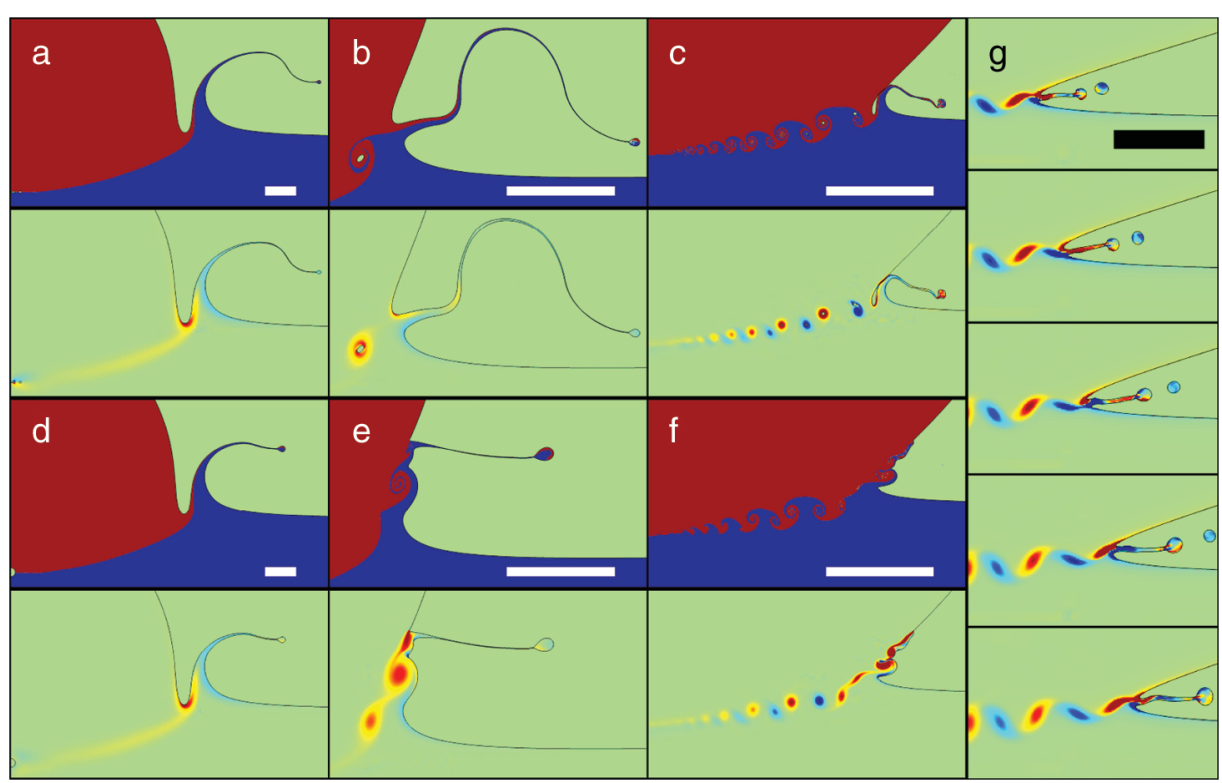

FIG. 4 (color). Vorticity structures during drop impact near the transition regime between a smooth ejecta sheet and irregular splashing. In the top images we differentiate the liquids originating from the drop (red) and from the pool (blue), from the air (green), as can be done in experiments by seeding one or the other with fluorescent dye [9]. The bottom images show the corresponding vorticity in the liquid. (a)-(c) Bumping transition, with $K=7.44 \times 10^{4}$, for increasing Reynolds numbers: Re $=1000$, 3552, and 14500 , respectively. (d)-(f) Quartering transition, with $K=3 \times 10^{4}$, for $\mathrm{Re}=1000,3552$, and 10000 , respectively. To allow direct comparison, the images of the first row (a)-(c) correspond to the same nondimensional time $\left(t^{*}=0.343,0.150\right.$, and 0.066 , respectively) as the ones in the second row (d)-(f), with the same field of view. (c) and (f) correspond to a water drop of $D=$ $4.6 \mathrm{~mm}$ impacting at $2.84 \mathrm{~m} / \mathrm{s}$ and $1.98 \mathrm{~m} / \mathrm{s}$, respectively. (g) Details of the early vortex shedding in the same case as (c), from $t^{*}=1.02 \times 10^{-2}$, and then a constant $\Delta t^{*}=4.5 \times 10^{-4}$. The period of this shedding shown here is approximately $3 \mu \mathrm{s}$, over a radial distance of $50 \mu \mathrm{m}$. The scale bars are $0.1 D$ long for (a)-(f), and $0.01 D$ long for $(\mathrm{g})$.

observe an unstable mode that involves both the jet and the vortex street [10]. During the early shedding [Fig. 4(g)], surface tension effects are higher because of the sharper surface geometry. As the angle of the neck increases, the amplitude of the oscillations increases. The ejecta can then climb on the drop at lower $K$ (f) or impact alternatively on the drop and the pool (c) in a similar way to the bumping, entrapping a row of bubble rings $[8,32]$. Four bubble rings can be clearly identified in Fig. 4(c), with a fifth one being created. Only well-resolved bubbles and droplets (larger area than 30 cells) are kept in the numerics, suggesting that smaller bubbles could be entrapped earlier.

From systematic experimental observations, reproduced with axisymmetric simulations, we have detailed a new mechanism explaining the irregular splashing of a water drop. Previously studied mechanisms have described the droplet separation from the rim of the ejecta $[8,33,34]$, or the destabilization of a liquid sheet $[35,36]$. Our mechanism, however, explains the breakup of the ejecta sheet by the destabilization of its base, through vortex shedding from the free surface.

The authors are grateful to A. Prosperetti and G. Agbaglah for comments, B. Marchand and A. Clo for assistance, T. El Sayed for sharing numerical resources, and $\mathrm{H}$. Pottmann for advice on curvature calculations. We also thank the GERRIS community for its support. M.-J. T. and S. T. T. were partly supported by KAUST-BERKELEY AEA Grant No. 7000000028.

*sigurdur.thoroddsen@kaust.edu.sa

[1] M. Rein, Fluid Dyn. Res. 12, 61 (1993).

[2] A. L. Yarin, Annu. Rev. Fluid Mech. 38, 159 (2006).

[3] A. M. Worthington, Proc. R. Soc. Lond. 34, 217 (1882).

[4] T. G. Etoh et al., IEEE Trans. Electron Devices 50, 144 (2003).

[5] S. T. Thoroddsen, T. G. Etoh, and K. Takehara, Annu. Rev. Fluid Mech. 40, 257 (2008).

[6] S. T. Thoroddsen, M.-J. Thoraval, K. Takehara, and T. G. Etoh, Phys. Rev. Lett. 106, 034501 (2011).

[7] L. V. Zhang, J. Toole, K. Fezzaa, and R. D. Deegan, J. Fluid Mech. 690, 5 (2012).

[8] D. A. Weiss and A. L. Yarin, J. Fluid Mech. 385, 229 (1999).

[9] S. T. Thoroddsen, J. Fluid Mech. 451, 373 (2002).

[10] See Supplemental Material at http://link.aps.org/ supplemental/10.1103/PhysRevLett.108.264506 for experimental details and video clips.

[11] C. D. Stow and M. G. Hadfield, Proc. R. Soc. A 373, 419 (1981).

[12] C. Mundo, M. Sommerfeld, and C. Tropea, Int. J. Multiph. Flow 21, 151 (1995) 
[13] G. Cossali, A. Coghe, and M. Marengo, Exp. Fluids 22, 463 (1997).

[14] C. Josserand and S. Zaleski, Phys. Fluids 15, 1650 (2003).

[15] G. Coppola, G. Rocco, and L. de Luca, Phys. Fluids 23, 022105 (2011).

[16] G. Tryggvason, R. Scardovelli, and S. Zaleski, Direct Numerical Simulations of Gas-Liquid Multiphase Flows (Cambridge University Press, Cambridge, England, 2011).

[17] S. Popinet, J. Comput. Phys. 228, 5838 (2009).

[18] G. Agbaglah et al., C.R. Mech. 339, 194 (2011).

[19] S. Popinet, GERRIS FLOW SOLVER, http://gfs.sf.net

[20] L. Xu, W. W. Zhang, and S. R. Nagel, Phys. Rev. Lett. 94, 184505 (2005).

[21] M. M. Driscoll and S. R. Nagel, Phys. Rev. Lett. 107, 154502 (2011).

[22] L. Duchemin and C. Josserand, Phys. Fluids 23, 091701 (2011).

[23] S. Mandre and M.P. Brenner, J. Fluid Mech. 690, 148 (2012).

[24] J. M. Kolinski, S. M. Rubinstein, S. Mandre, M.P. Brenner, D. A. Weitz, and L. Mahadevan, Phys. Rev. Lett. 108, 074503 (2012).
[25] R. C. A. van der Veen, T. Tran, D. Lohse, and C. Sun, Phys. Rev. E 85, 026315 (2012).

[26] G. K. Batchelor, An Introduction to Fluid Dynamics (Cambridge University Press, Cambridge, England, 1967).

[27] B. Peck and L. Sigurdson, Phys. Fluids 6, 564 (1994).

[28] R. W. Cresswell and B. R. Morton, Phys. Fluids 7, 1363 (1995).

[29] D. Yu and G. Tryggvason, J. Fluid Mech. 218, 547 (1990).

[30] S. D. Howison, J. R. Ockendon, J. M. Oliver, R. Purvis, and F. T. Smith, J. Fluid Mech. 542, 1 (2005).

[31] C. H. K. Williamson and R. Govardhan, Annu. Rev. Fluid Mech. 36, 413 (2004).

[32] M. R. Davidson, Chem. Eng. Sci. 57, 3639 (2002).

[33] D. Gueyffier and S. Zaleski, C. R. Acad. Sci. IIb Mec. 326, 839 (1998).

[34] L. V. Zhang, P. Brunet, J. Eggers, and R. D. Deegan, Phys. Fluids 22, 122105 (2010).

[35] N. Dombrowski and W. R. Johns, Chem. Eng. Sci. 18, 203 (1963).

[36] E. Villermaux and C. Clanet, J. Fluid Mech. 462, 341 (2002). 